\title{
Associations between perceived friends' support of healthy eating and meal skipping in adolescence
}

\author{
Sharon Rosenrauch, Kylie Ball and Karen E Lamb* \\ Institute for Physical Activity and Nutrition (IPAN), School of Exercise and Nutrition Sciences, Deakin University, \\ 221 Burwood Highway, Burwood, Geelong, VIC 3125, Australia
}

Submitted 27 October 2016: Final revision received 29 June 2017: Accepted 26 July 2017: First published online 7 September 2017

\begin{abstract}
Objective: Meal skipping is a relatively common behaviour during adolescence. As peer influence increases during adolescence, friendship groups may play a role in determining eating patterns such as meal skipping. The current study examined cross-sectional and longitudinal associations between perceived friends' support of healthy eating and breakfast and lunch skipping among adolescents.

Design: Survey of intrapersonal, social and environmental factors that may influence eating patterns at baseline (2004/05) and follow-up (2006/07).

Setting: Thirty-seven secondary schools in Victoria, Australia.

Subjects: Sample of 1785 students aged 12-15 years at baseline.

Results: Adolescents who reported that their friends sometimes or often ate healthy foods with them were less likely (adjusted OR; 95\% CI) to skip breakfast (sometimes: $0.71 ; 0.57,0.90$; often: $0.54 ; 0.38,0.76$ ) or lunch (sometimes: 0.61 ; 0.41, 0.89; often: $0.59 ; 0.37,0.94$ ) at baseline than those who reported their friends never or rarely displayed this behaviour. Although this variable was associated with lunch skipping at follow-up, there was no evidence of an association with breakfast skipping at follow-up. There was no evidence of an association between perceived encouragement of healthy eating, and an inconsistent relationship between perceived discouragement of junk food consumption, and meal skipping.

Conclusions: Friends eating healthy foods together may serve to reduce meal skipping during early adolescence, possibly due to the influence of directly observable behaviour and shared beliefs held by those in the same friendship group. Verbal encouragement or discouragement from friends may be less impactful an influence on meal skipping (than directly observable behaviours) in adolescents.
\end{abstract}

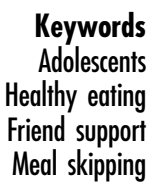

Keywords

Healthy eating Meal skipping

\begin{abstract}
Adolescents are a population particularly at risk of nutritional deficiencies due to their increased micronutrient needs for growth, as well as their changing lifestyle and eating habits arising from increased independence ${ }^{(1)}$. Thus, regular meal consumption is imperative for optimal health and development during this life stage ${ }^{(2)}$. However, skipping meals is a relatively frequent behaviour during middle and late adolescence ${ }^{(3)}$, and this behaviour has been found to have increased in recent years ${ }^{(4,5)}$. Despite reporting being aware of its adverse health effects, adolescents are increasingly viewing meal skipping as a quick and effective method for weight loss ${ }^{(5)}$. Breakfast is typically the most commonly reported missed meal ${ }^{(6-9)}$, perhaps explaining why the bulk of existing meal skipping literature has tended to focus on factors that influence breakfast omission ${ }^{(10-13)}$.
\end{abstract}

Unfortunately, nutrient deficiencies associated with meal (particularly breakfast) omission do not appear to be compensated for by dietary changes throughout other times of the day ${ }^{(14)}$. In fact, adolescents who frequently skip breakfast have been found to be more likely to also skip other meals ${ }^{(15)}$. This is concerning since adolescence is the period in which long-term eating habits are established $^{(16)}$ and meal skipping during adolescence can lead to poor health outcomes, such as compromised nutritional status $^{(14,17)}$, as well as long-term health consequences such as higher BMI and impaired cardiometabolic health $^{(18)}$.

Adolescents' eating patterns are potentially influenced by a multitude of factors, including personal characteristics such as body weight, lifestyle and psychological factors, and macro systems such as mass media and advertising ${ }^{(2)}$. 
Socio-environmental factors, including the adolescent's family unit, peer and friendship groups, have also been shown to play a role in determining eating behaviours $^{(3,19)}$. Pearson et al. ${ }^{(20)}$ investigated associations between maternal behaviour and adolescent meal skipping, finding maternal meal skipping to be a significant predictor of adolescent meal omission. However, that study examined social influences only in the form of potentially maladaptive behaviours (i.e. maternal meal skipping or weight watching) as potential predictors of meal-skipping behaviour. Associations between positive health behaviours, such as healthy eating, and meal skipping were not studied. Further, as an adolescent ages and spends less time at home there is a gradual shift in reliance from family to peers and thus the influence of friends becomes increasingly salient ${ }^{(3)}$. Adolescents have reported that the behaviours, attitudes and especially encouragement from friends influence their food choices and eating patterns ${ }^{(21-24)}$. Therefore, it is possible that identifying important peer-related predictors of adolescent meal skipping may assist in developing nutrition interventions to curtail this unhealthful behaviour.

A number of studies have examined the relationship between meal skipping and unhealthy dietary outcomes ${ }^{(20,23-25)}$. For example, the Australian Youth Eating Patterns (YEP) study ${ }^{(20)}$ examined peer-related predictors of adolescent meal skipping as a single outcome variable (i.e. not as part of a combined measure such as 'unhealthy dietary practices'). Results from that study suggested that adolescents were more likely to skip meals if they perceived that their best friend skipped meals, although these associations were found for all meals for girls but only for lunch skipping for boys.

The objective of the current research was to expand on previous findings from the YEP study, reanalysing data presented elsewhere ${ }^{(20,26)}$ to consider broader peer influences, rather than only maternal and best friend influences, on meal skipping. While associations between maladaptive behaviours of others, such as meal skipping and weight watching, have been assessed, no studies have examined associations between positive health behaviours of peers and meal skipping in adolescents. The present study considers positive peer support influences since these, if found to be associated with meal skipping, provide greater practical utility for interventions, given that promoting healthy eating may be easier than correcting a maladaptive behaviour. The longitudinal nature of the YEP study provides an advantage over most studies of meal skipping which are cross-sectional, in that it enables assessment of whether influences in early adolescence are associated with meal skipping in later adolescence. Therefore, the present study aimed to examine: (i) if perceived peer support of healthy eating in early adolescence was associated with meal skipping during this time; and (ii) if perceived peer support of healthy eating in early adolescence was associated with meal skipping in later adolescence.

\section{Methods}

\section{Participants and setting}

Data were drawn from the YEP study, an online food habits survey of adolescents from Victoria, Australia. Details of the YEP study procedures have been described elsewhere ${ }^{(27-29)}$. Briefly, a sample of 9842 students in Year 7 (aged 12-13 years) and Year 9 (aged 14-15 years) from thirty-seven participating schools were invited to complete the online survey during 2004-2005 (baseline). In total, 3264 adolescents ( $n 2010$ in Year 7, n 1254 in Year 9; response rate $=33 \%$ of those invited) completed the survey at baseline. In 2006-2007 (follow-up), 1938 adolescents participated in the study, representing a response rate of $59 \%$ of the baseline sample.

To be eligible for inclusion in the present study, adolescents had to have participated in both waves of data collection and provided data on all variables of interest. The final sample in this complete case analysis consisted of 1785 adolescents ( $92 \%$ of those who completed followup). The characteristics of this sample were compared with those of the baseline sample (see online supplementary material, Supplemental Table 1). Characteristics were similar for both, although fewer students were from rural schools (25\%) in the follow-up study compared with baseline (32\%).

The study was approved by the Ethics Committee of Deakin University, the Victorian Department of Education and Training and the Catholic Education Office (EC 227-2003).

\section{Measures}

The YEP study collected information on demographic characteristics of respondents including age, school year (Year 7 or Year 9 at baseline), sex and region of residence (metropolitan or rural).

\section{Outcome variables}

Meal skipping was assessed by asking respondents how often over the past month they had 'skipped breakfast', 'skipped lunch' and/or 'skipped dinner'. In the present study, dinner skipping was omitted from further analysis due to the small number of students (only $4 \%$ ) reporting dinner skipping, consistent with past research ${ }^{(10,12)}$. Responses to breakfast and lunch skipping included 'not in the last month', 'once/twice a month', 'once/twice a week', 'most days' and 'every day'. In the current study, consistent with previous approaches ${ }^{(20,26,27)}$, adolescents were categorized as 'frequent skippers' if they reported skipping meals on 'most days' or 'every day' and 'infrequent skippers' otherwise.

\section{Predictor variables}

Three survey items assessed perceived friends' level of support for healthy eating and were adapted from a previously validated measure of social support for diet and exercise behaviours ${ }^{(30)}$. Respondents were asked to rate how 
frequently over the past year their friends '(ate) healthy foods with (them)', 'encouraged (them) not to eat "junk" food (they) felt like' and 'encouraged (them) to eat healthy foods'. Response options were 'never/rarely', 'sometimes' and 'often'.

\section{Confounders}

Prior research has demonstrated that sociodemographic factors including $\operatorname{sex}^{(8,10,31,32)}$, age ${ }^{(4,8,33)}$ and region of residence ${ }^{(13,34)}$ may influence adolescent meal-skipping behaviour (outcome variable) and sensitivity to peer support (predictor variables). Thus, these variables, measured at baseline, were examined as potential confounders of the association between peer support and meal skipping.

\section{Maternal and best friend influences}

Pearson et al. considered both maternal and best friend influences on meal skipping in $\mathrm{YEP}^{(20)}$. These included the following variables: mother often skips meals ('yes' or 'no/ unsure'), mother watches her weight ('yes' or 'no/unsure'), mother models healthy eating ('low' or 'high'), best friend often skips meals ('yes' or 'no/unsure'), best friend watches her weight ('yes' or 'no/unsure') and best friend models healthy eating ('low' or 'high').

\section{Statistical analysis}

Descriptive statistics were calculated to describe meal skipping, peer support and the sociodemographic characteristics of participants at baseline and follow-up. To address the first aim, separate logistic regression models were fitted to examine associations between each of the three variables related to perceived friends' support of healthy eating at baseline and each of the meal-skipping outcomes at baseline. Unadjusted models were examined, as well as models adjusting for potential confounders. To address the second aim, similar models were fitted for meal-skipping outcomes at follow-up, considering the baseline predictors, adjusting for meal skipping at baseline. All models adjusted for the sampling of students from within schools using robust clustered SE. In sensitivity analyses, the maternal and best friend influences on meal skipping considered by Pearson et al. ${ }^{(20)}$ were included in the adjusted models to determine if this affected the estimated associations. Finally, models were fitted to examine the cross-sectional association between perceived friends' support at follow-up and meal skipping at follow-up. Descriptive statistics were conducted using the statistical software package IBM SPSS Statistics version 21.0 (2012) and regression models were fitted in the statistical software package Stata version 14 (2015).

\section{Results}

Descriptive statistics are presented in Table 1. At baseline, the adolescent sample consisted predominantly of Year 7
Table 1 Summary statistics for those who completed both baseline and follow-up surveys ( $N$ 1785); adolescents from thirty-seven secondary schools in Victoria, Australia, Youth Eating Patterns study

\begin{tabular}{|c|c|c|}
\hline Measure & $n$ & $\%$ \\
\hline \multicolumn{3}{|l|}{ Outcome variables $\dagger$} \\
\hline \multicolumn{3}{|l|}{ Skips breakfast } \\
\hline \multicolumn{3}{|l|}{ Frequent skippers } \\
\hline Baseline (2004/05) & 334 & $18 \cdot 7$ \\
\hline Follow-up (2006/07) & 413 & 23.1 \\
\hline \multicolumn{3}{|l|}{ Skips lunch } \\
\hline \multicolumn{3}{|l|}{ Frequent skippers } \\
\hline Baseline (2004/05) & 193 & $10 \cdot 8$ \\
\hline Follow-up (2006/07) & 177 & 9.9 \\
\hline \multicolumn{3}{|l|}{ Predictor variables } \\
\hline \multicolumn{3}{|l|}{ Friends eat healthy foods with you } \\
\hline Never/rarely & 512 & $28 \cdot 7$ \\
\hline Sometimes & 807 & $45 \cdot 2$ \\
\hline Often & 466 & $26 \cdot 1$ \\
\hline \multicolumn{3}{|c|}{ Friends encourage you not to eat 'junk' food } \\
\hline Never/rarely & 1116 & 62.5 \\
\hline Sometimes & 507 & 28.4 \\
\hline Often & 162 & $9 \cdot 1$ \\
\hline \multicolumn{3}{|c|}{ Friends encourage you to eat healthy foods } \\
\hline Never/rarely & 972 & 54.5 \\
\hline Sometimes & 557 & $31 \cdot 2$ \\
\hline Often & 256 & $14 \cdot 3$ \\
\hline \multicolumn{3}{|l|}{ Sociodemographic characteristics } \\
\hline \multicolumn{3}{|l|}{ Sex } \\
\hline Girls & 996 & $55 \cdot 8$ \\
\hline Boys & 789 & $44 \cdot 2$ \\
\hline \multicolumn{3}{|l|}{ School year } \\
\hline Year 7 (Year 9 at follow-up) & 1155 & $64 \cdot 7$ \\
\hline Year 9 (Year 11 at follow-up) & 630 & $35 \cdot 3$ \\
\hline \multicolumn{3}{|l|}{ Region } \\
\hline Metropolitan & 1335 & 74.8 \\
\hline Rural & 450 & $25 \cdot 2$ \\
\hline
\end{tabular}

†Skipping meals: adolescents were classified as 'frequent skippers' (of breakfast, lunch) if they reported skipping meals on 'most days' or 'every day', and 'infrequent' skippers if they reported otherwise.

students (65\%; mean age 13.4 years) residing in metropolitan areas (75\%). A higher number of girls (56\%) than boys participated in both waves of the YEP study. Breakfast was the most commonly reported missed meal, with a higher frequency of participants reporting frequent breakfast skipping at baseline (18.7\%) and follow-up $(23 \cdot 1 \%)$ compared with lunch skipping (10.8\% at baseline; $9.9 \%$ at follow-up). Overall, $60 \%$ of adolescents who reported frequent breakfast skipping at baseline also reporting frequent breakfast skipping at follow-up, while only $27 \%$ of adolescents who reported frequent lunch skipping at baseline reported lunch skipping at follow-up (see online supplementary material, Supplemental Tables 2 and 3).

\section{Associations between perceived friends' support of bealthy eating and meal skipping}

Associations between perceived friends' support of healthy eating and meal skipping at baseline

Only one of the measures of perceived friends' support of healthy eating was found to be associated with the frequency of meal skipping at baseline: frequency of friends 
Table 2 Associations between perceived friends' support of healthy eating and frequency of breakfast and lunch skipping at baseline $(2004 / 05)$ among adolescents $(N$ 1785) from thirty-seven secondary schools in Victoria, Australia, Youth Eating Patterns study

\begin{tabular}{|c|c|c|c|c|c|c|c|c|}
\hline \multirow[b]{3}{*}{ Measure } & \multicolumn{4}{|c|}{ Breakfast skipping } & \multicolumn{4}{|c|}{ Lunch skipping } \\
\hline & \multicolumn{2}{|c|}{ Unadjusted $†$} & \multicolumn{2}{|c|}{ Adjusted $\ddagger$} & \multicolumn{2}{|c|}{ Unadjusted $†$} & \multicolumn{2}{|c|}{ Adjusted $\ddagger$} \\
\hline & OR & $95 \% \mathrm{Cl}$ & AOR & $95 \% \mathrm{Cl}$ & OR & $95 \% \mathrm{Cl}$ & AOR & $95 \% \mathrm{Cl}$ \\
\hline \multicolumn{9}{|c|}{ Friends eat healthy food with you§ } \\
\hline Sometimes & $0.75^{\star}$ & $0.60,0.99$ & $0.71^{\star *}$ & $0.57,0.90$ & $0.62^{*}$ & $0.42,0.90$ & $0.61^{\star *}$ & $0.41,0.89$ \\
\hline Often & $0.60^{* *}$ & $0.43,0.83$ & $0.54^{\star \star \star}$ & $0.38,0.76$ & $0.60^{*}$ & $0.37,0.97$ & $0.59^{*}$ & $0.37,0.94$ \\
\hline Sex\| & & & $1.77^{\star \star \star}$ & $1 \cdot 39,2 \cdot 26$ & & & 1.28 & $0.89,1.84$ \\
\hline Year level\| & & & 1.23 & $0.94,1.61$ & & & 1.25 & $0.82,1.90$ \\
\hline Region\| & & & 0.80 & $0.55,1.14$ & & & 0.79 & $0.54,1.17$ \\
\hline \multicolumn{9}{|c|}{ Friends discourage 'junk' food } \\
\hline Sometimes & 0.93 & $0.70,1.25$ & 0.92 & $0.69,1.22$ & 0.96 & $0.76,1.23$ & 0.97 & $0.76,1.23$ \\
\hline Often & 0.88 & $0.58,1.34$ & 0.89 & $0.58,1.35$ & 0.69 & $0.37,1.29$ & 0.70 & $0.38,1.29$ \\
\hline Sex & & & $1.63^{\star \star *}$ & $1.30,2.05$ & & & 1.19 & $0.84,1.69$ \\
\hline Year level & & & $1.28^{*}$ & $0.98,1.68$ & & & 1.29 & $0.86,1.93$ \\
\hline Region & & & 0.79 & $0.54,1.15$ & & & 0.78 & $0.52,1.17$ \\
\hline \multicolumn{9}{|c|}{ Friends encourage healthy food } \\
\hline Sometimes & 1.07 & $0.80,1.42$ & 1.03 & $0.77,1.38$ & 0.89 & $0.63,1.27$ & 0.89 & $0.62,1.27$ \\
\hline Often & 1.09 & $0.76,1.57$ & 1.08 & $0.74,1.57$ & 0.89 & $0.63,1.24$ & 0.90 & $0.64,1.28$ \\
\hline Sex & & & $1.62^{\star \star \star}$ & $1 \cdot 29,2.03$ & & & 1.20 & $0.84,1.71$ \\
\hline Year level & & & 1.30 & $0.98,1.71$ & & & 1.29 & $0.85,1.94$ \\
\hline Region & & & 0.79 & $0.54,1.15$ & & & 0.78 & $0.53,1.16$ \\
\hline
\end{tabular}

${ }^{*} P<0.05 ;{ }^{* \star} P<0.01 ;{ }^{* \star *} P<0.001$.

†Unadjusted logistic regression analyses of the association between each perceived friends' support of healthy eating measure and each of the meal-skipping outcomes at baseline, with clustered SE to account for clustering within school. The Hosmer and Lemeshow goodness-of-fit statistic was acceptable for all unadjusted baseline models (all test statistics $(\mathrm{df}=6, n 1785)<7.18$, all $P>0.305$ ).

$\ddagger$ Adjusted logistic regression analyses of the association between each perceived friends' support of healthy eating measure and each of the meal-skipping outcomes at baseline adjusting for adolescent sex, school year and region of residence, with clustered SE to account for clustering within school.

$\S N$ Never/rarely was used as the reference category for perceived friend support predictor variables in all models.

||Reference categories: male, Year 7, Metropolitan.

eating healthy food with them (Table 2). The results suggested that adolescents who reported that their friends sometimes ate healthy food with them had lower odds of reporting frequent breakfast skipping (adjusted odds ratio $(\mathrm{AOR})=0.71 ; 95 \% \mathrm{CI} 0.57,0.90)$ and frequent lunch skipping (AOR $=0.61 ; 95 \% \mathrm{CI} 0.41,0.89)$ at baseline when compared with their peers who reported their friends never or rarely ate healthy foods with them. In addition, the odds of frequent breakfast or lunch skipping were lower for adolescents who reported their friends often ate healthy foods with them at baseline $(\mathrm{AOR}=0.54 ; 95 \% \mathrm{CI}$ $0.38,0.76$ and $\mathrm{AOR}=0.59 ; 95 \% \mathrm{CI} 0.37,0.94$, respectively) compared with those who reported their friends never or rarely ate healthy foods with them. In general, these associations remained after adjustment for either maternal influences or best friend influences (or both) on meal skipping, although estimated AOR were closer to 1 after accounting for best friend influences (see online supplementary material, Supplemental Table 4). Furthermore, there was no evidence of a difference in odds of lunch skipping for those whose friends often ate healthy foods with them and those whose friends never or rarely ate healthy foods with them after accounting for best friend influences.

In summary, these results suggest that the higher the level of perceived friend support of healthy eating, the lower the odds of breakfast and/or lunch skipping at baseline.
Associations between perceived friends' support of healthy eating and meal skipping at follow-up

Despite being found to be associated with the frequency of breakfast skipping at baseline, there was no evidence of an association between friends eating healthy food with them at baseline and frequency of breakfast skipping at follow-up (Table 3). Cross-sectional analysis at the followup time point showed similar associations to that of the cross-sectional baseline analysis (Table 4). There was no evidence of a difference in the odds of lunch skipping between those who reported that their friends often ate healthy foods with them compared with those who reported that their friends never ate healthy food with them $(\mathrm{AOR}=0.76 ; 95 \% \mathrm{CI} 0.50,1.53)$, although there was evidence of lower odds of frequent lunch skipping among those whose friends sometimes ate healthy foods with them $(\mathrm{AOR}=0.72 ; 95 \% \mathrm{CI} 0.46,0.96)$. This finding was consistent with cross-sectional analyses at follow-up (Table 4).

Although not found to be associated with meal skipping at baseline, there was some evidence of an association between friends sometimes discouraging junk food consumption at baseline and breakfast skipping at follow-up $(\mathrm{AOR}=1.58 ; 95 \% \mathrm{CI} 1.21,2.07)$ but not lunch skipping (Table 3). Evidence of an association remained after adjustment for best friend influences (AOR $=1.52 ; 95 \% \mathrm{CI}$ $1 \cdot 17,1 \cdot 98$ ) or maternal influences (AOR: 1.56; $95 \%$ CI 1.18, $2.05)$ as shown in the online supplementary material, 
Table 3 Associations between perceived friends' support of healthy eating and frequency of breakfast and lunch skipping at follow-up $(2006 / 07)$ among adolescents $(N$ 1785) from thirty-seven secondary schools in Victoria, Australia, Youth Eating Patterns study

\begin{tabular}{|c|c|c|c|c|c|c|c|c|}
\hline \multirow[b]{3}{*}{ Measure } & \multicolumn{4}{|c|}{ Breakfast skipping } & \multicolumn{4}{|c|}{ Lunch skipping } \\
\hline & \multicolumn{2}{|c|}{ Unadjusted $\dagger$} & \multicolumn{2}{|c|}{ Adjusted $\ddagger$} & \multicolumn{2}{|c|}{ Unadjusted† } & \multicolumn{2}{|c|}{ Adjusted $\ddagger$} \\
\hline & OR & $95 \% \mathrm{Cl}$ & AOR & $95 \% \mathrm{Cl}$ & OR & $95 \% \mathrm{Cl}$ & AOR & $95 \% \mathrm{Cl}$ \\
\hline \multicolumn{9}{|c|}{ Friends eat healthy food with you§ } \\
\hline Sometimes & 0.86 & $0.70,1 \cdot 10$ & 0.93 & $0.74,1.16$ & $0.68^{*}$ & $0.48,0.96$ & $0.72^{*}$ & $0.46,0.96$ \\
\hline Often & 0.83 & $0.63,1.10$ & 0.95 & $0.70,1.28$ & 0.79 & $0.52,1.22$ & 0.76 & $0.50,1.53$ \\
\hline Sex\| & & & $1.44^{\star \star \star}$ & $1.16,1.78$ & & & 1.23 & $0.89,1.69$ \\
\hline Year level $\|$ & & & 1.11 & $0.89,1.39$ & & & 0.99 & $0.71,1.37$ \\
\hline Region\| & & & 0.89 & $0.70,1.12$ & & & 0.74 & $0.50,1.08$ \\
\hline \multicolumn{9}{|c|}{ Friends discourage 'junk' food } \\
\hline Sometimes & $1.44^{* *}$ & $1.14,1.81$ & $1.58^{\star \star}$ & $1.21,2.07$ & 0.99 & $0.61,1.60$ & 0.98 & $0.60,1.59$ \\
\hline Often & 1.07 & $0.72,1.57$ & $1 \cdot 15$ & $0.75,1.75$ & 0.99 & $0.57,1.73$ & 1.01 & $0.57,1.99$ \\
\hline Sex & & & $1.39^{* *}$ & $1.13,1.71$ & & & 1.15 & $0.80,1.66$ \\
\hline Year level & & & $1 \cdot 15$ & $0.91,1.45$ & & & 0.94 & $0.55,1.61$ \\
\hline Region & & & 0.90 & $0.71,1.14$ & & & 0.76 & $0.49,1.17$ \\
\hline \multicolumn{9}{|c|}{ Friends encourage healthy food } \\
\hline Sometimes & 1.22 & $0.98,1.51$ & 1.20 & $0.98,1.46$ & 0.82 & $0.51,1.32$ & 0.82 & $0.49,1.35$ \\
\hline Often & 1.34 & $0.96,1.86$ & 1.35 & $1.00,1.81$ & 0.96 & $0.57,1.62$ & 0.97 & $0.60,1.58$ \\
\hline Sex & & & $1.39^{\star \star}$ & $1.13,1.72$ & & & $1 \cdot 16$ & $0.82,1.66$ \\
\hline Year level & & & 1.14 & $0.91,1.43$ & & & 0.93 & $0.55,1.58$ \\
\hline Region & & & 0.88 & $0.70,1.18$ & & & 0.75 & $0.49,1.16$ \\
\hline
\end{tabular}

${ }^{\star} P<0.05 ;{ }^{* *} P<0.01 ;{ }^{* \star} P<0.001$.

tUnadjusted logistic regression analyses of the association between each perceived friends' support of healthy eating measure and each of the meal-skipping outcomes at follow-up, with clustered SE to account for clustering within school. The Hosmer and Lemeshow goodness-of-fit statistic was acceptable for all unadjusted baseline models (all test statistics $(\mathrm{df}=6, n 1785)<8.97$, all $P>0.175)$.

$\ddagger$ Adjusted logistic regression analyses of the association between each perceived friends' support of healthy eating measure and each of the meal-skipping outcomes at follow-up adjusting for baseline meal skipping, adolescent sex, school year and region of residence, with clustered SE to account for clustering within school.

$\S N$ ever/rarely was used as the reference category for perceived friend support predictor variables in all models.

||Reference categories: male, Year 7, Metropolitan.

Table 4 Cross-sectional associations between perceived friends' support of healthy eating and frequency of breakfast and lunch skipping at follow-up (2006/07) among adolescents $(N$ 1785) from thirty-seven secondary schools in Victoria, Australia, Youth Eating Patterns study

\begin{tabular}{|c|c|c|c|c|c|c|c|c|}
\hline \multirow[b]{3}{*}{ Measure } & \multicolumn{4}{|c|}{ Breakfast skipping } & \multicolumn{4}{|c|}{ Lunch skipping } \\
\hline & \multicolumn{2}{|c|}{ Unadjusted† } & \multicolumn{2}{|c|}{ Adjusted $\neq$} & \multicolumn{2}{|c|}{ Unadjusted $†$} & \multicolumn{2}{|c|}{ Adjusted $\ddagger$} \\
\hline & OR & $95 \% \mathrm{Cl}$ & AOR & $95 \% \mathrm{Cl}$ & OR & $95 \% \mathrm{Cl}$ & AOR & $95 \% \mathrm{Cl}$ \\
\hline \multicolumn{9}{|c|}{ Friends eat healthy food with you§ } \\
\hline Sometimes & $0.75^{\star}$ & $0.59,0.96$ & $0.71^{\star \star}$ & $0.57,0.89$ & 0.69 & $0.47,1.00$ & 0.68 & $0.47,0.99$ \\
\hline Often & 0.70 & $0.51,0.97^{\star}$ & $0.60^{\star \star}$ & $0.43,0.84$ & $0.42^{\star \star \star}$ & $0.28,0.63$ & $0.39^{\star \star \star}$ & $0.25,0.61$ \\
\hline Sex\| & & & $1.74^{\star \star \star}$ & $1 \cdot 44,2 \cdot 11$ & & & 1.36 & $0.92,2.01$ \\
\hline Year" level\| & & & 1.22 & $1.00,1.50$ & & & 1.00 & $0.61,1.65$ \\
\hline Region\| & & & 0.85 & $0.67,1.06$ & & & 0.75 & $0.47,1.19$ \\
\hline \multicolumn{9}{|c|}{ Friends discourage 'junk' food } \\
\hline Sometimes & $1.27^{\star}$ & $1.02,1.60$ & 1.25 & $1.00,1.56$ & 0.89 & $0.70,1.15$ & 0.89 & $0.69,1.13$ \\
\hline Often & 1.05 & $0.76,1.44$ & 1.03 & $0.75,1.40$ & 0.84 & $0.52,1.34$ & 0.83 & $0.51,1.34$ \\
\hline Sex & & & $1.61^{\star \star *}$ & $1.33,1.94$ & & & $1 \cdot 19$ & $0.84,1.69$ \\
\hline Year level & & & 1.20 & $0.97,1.49$ & & & 1.00 & $0.60,1.69$ \\
\hline Region & & & 0.83 & $0.65,1.06$ & & & 0.73 & $0.46,1.17$ \\
\hline \multicolumn{9}{|c|}{ Friends encourage healthy food } \\
\hline Sometimes & 1.13 & $0.92,1.38$ & 1.07 & $0.88,1.29$ & 1.07 & $0.73,1.55$ & 1.05 & $0.72,1.53$ \\
\hline Often & 1.36 & $1.05,1.76^{\star}$ & 1.26 & $0.98,1.63$ & 1.01 & $0.65,1.59$ & 0.98 & $0.60,1.59$ \\
\hline Sex & & & 1.58 & $1.32,1.90$ & & & 1.18 & $0.82,1.70$ \\
\hline Year level & & & 1.21 & $0.97,1.51$ & & & 1.00 & $0.59,1.69$ \\
\hline Region & & & 0.84 & $0.66,1.06$ & & & 0.73 & $0.46,1.17$ \\
\hline
\end{tabular}

${ }^{\star} P<0.05 ;{ }^{* *} P<0.01 ;{ }^{* \star} P<0.001$.

+Unadjusted logistic regression analyses of the association between each perceived friends' support of healthy eating measure and each of the meal-skipping outcomes at follow-up, with clustered SE to account for clustering within school. The Hosmer and Lemeshow goodness-of-fit statistic was acceptable for all unadjusted models (all test statistics ( $\mathrm{df}=6, n 1785)<7 \cdot 18$, all $P>0.305$ ).

$\ddagger$ Adjusted logistic regression analyses of the association between each perceived friends' support of healthy eating measure and each of the meal-skipping outcomes at follow-up adjusting for adolescent sex, school year and region of residence, with clustered SE to account for clustering within school.

$\S N e v e r /$ rarely was used as the reference category for perceived friend support predictor variables in all models.

||Reference categories: male, Year 9, Metropolitan. 
Supplemental Table 5. This association was also evident in unadjusted cross-sectional follow-up analysis (Table 4) but attenuated in adjusted analysis (AOR $=1 \cdot 25 ; 95 \%$ CI 1.00 , 1.56). These results are consistent with a hypothesis that increased perceived friend support would be associated with less frequent meal skipping. However, there was no evidence of a difference in frequency of breakfast skipping for those whose friends often discouraged junk food consumption compared with never in either the longitudinal or cross-sectional follow-up analysis (Tables 3 and 4).

There was no evidence of an association between friends encouraging healthy food consumption and breakfast or lunch skipping at follow-up in adjusted longitudinal (Table 3) or cross-sectional (Table 4) analyses.

\section{Discussion}

The aim of the present study was to examine whether perceived friends' support of healthy eating was associated with frequent breakfast and lunch skipping in a sample of Australian adolescents. The study analysed data previously examined by Pearson et al. ${ }^{(20)}$, to examine whether maternal and best friend influence as a predictor of meal skipping would extend more generally to broader friendship groups, as it is likely that the behaviour of more than one friend will have a greater impact on behaviour. Further, maternal presence may be non-existent (e.g. single-parent households), while friendship presence is more probable. It was hypothesized that adolescents who reported greater levels of perceived friend support (measured as having friends eat healthy foods with them, discourage the consumption of junk food and encourage the consumption of healthy foods) would be less likely to report meal skipping at baseline and two years later at follow-up.

Friends' healthy eating (as opposed to meal skipping) behaviour was examined as a potential predictor of meal skipping due to the practical utility of promoting healthy eating $v$. correcting a maladaptive behaviour in a school and government policy context. Further, best friend meal skipping behaviour was previously examined in this group of adolescents, with results showing increased odds of meal skipping among adolescents whose best friend skipped meals. The results from the analysis of meal skipping frequency at baseline in the present study provided partial support for the first hypothesis, that higher levels of perceived friend support at baseline would be associated with less frequent meal skipping at baseline. Only one aspect of perceived friend support considered in the present study was found to be associated with breakfast and lunch skipping at baseline, with adolescents who reported that their friends ate healthy foods with them found to have lower odds of frequent breakfast or lunch skipping. This corresponds with other evidence suggesting that the presence of peers and friends at eating occasions increases adolescents' energy intake and likelihood of meal and snack consumption ${ }^{(25,35)}$. Friendship group's breakfast intake has been found to be positively associated with adolescent breakfast eating ${ }^{(36)}$. This is significant given the known health benefits of regular breakfast consumption and the fact that breakfast consumption is generally low among adolescents ${ }^{(14)}$. Intervention strategies that have applied school breakfast programmes such as grab-n-go breakfasts and social marketing programmes have been found to increase breakfast consumption while promoting social norms and interactions, particularly among adolescent girls. For adolescents and their friendship groups, consuming breakfast at school could be promoted as a social opportunity ${ }^{(36)}$. While the exact mechanism accounting for the effects of peer influences on healthful and frequent eating remains unclear, some studies suggest this effect may be due to modelling, particularly during early adolescence ${ }^{(23-25,37)}$. Although modelling was not directly assessed in the present study, it is interesting to reflect upon its potential importance when examining peer influence on eating behaviours.

Modelling regular healthy food consumption by friends may reduce meal skipping in adolescence via a systematic matching of eating patterns ${ }^{(25)}$. Adolescents who witness their friends consuming healthy foods are more likely to mimic this behaviour in an attempt to conform and/or integrate themselves to others ${ }^{(25)}$. This may in turn reduce adolescents' likelihood of meal skipping on occasions for which their friend is present, such as during lunch at school. Adolescents who witness their friends eating healthy foods may also follow suit and consume meals (reducing their likelihood of skipping) in an attempt to create a good impression and avoid the stigma incurred by overweight individuals who eat 'unhealthy' foods ${ }^{(25)}$. Indeed, perceived peer support for healthy eating has been found to be a negative longitudinal predictor of later fast-food intake ${ }^{(38)}$. This fear of being associated with traits of overweight individuals may be especially true for adolescent girls and during late adolescence ${ }^{(39)}$, possibly explaining why friends eating healthy foods also emerged as a significant predictor of lunch skipping at follow-up.

While the mechanisms described above are just a few that could potentially explain associations between this type of perceived friend support and lunch skipping, it is interesting to note that adolescents who reported that their friends supported their healthy eating by consuming healthy foods with them were less likely to skip breakfast at baseline, a meal for which their friends are unlikely to be present. This is perhaps unsurprising given research has demonstrated adolescent friendship groups often share similar beliefs and behaviours surrounding food $^{(21-24)}$. Thus, it is likely that adolescents who have friends who demonstrate healthy eating behaviours 
maintain healthy eating beliefs and habits themselves ${ }^{(20)}$. In this instance, it appears that adolescents may not only come to adopt the behaviours their friends model, but may also pick up some of their friends' related nutrition beliefs and values, which may act to influence their own eating behaviours even when their friends are absent.

Although the present study found that having friends who concurrently ate healthy food was negatively associated with meal skipping, there was no evidence of an association between friends' general encouragement of healthy eating and adolescent meal skipping. These findings resemble those of a previous study involving YEP data which found that adolescents who witnessed their best friend skipping meals were more likely to skip themselves but that adolescents who reported their best friend tended to 'watch their weight' were no more or less likely to skip ${ }^{(20)}$. This result suggests that modelling behaviour may be the more important mechanism influencing adolescent meal skipping. Combined with these earlier findings ${ }^{(20)}$, the present results suggest adolescents may be more able to identify specific, obvious behaviour strategies in their friends (in this context, observable healthy eating behaviours) and adopt these behaviours themselves to a greater extent than when they receive more general dietary encouragement.

Although there was some evidence to support the hypothesis that increased perceived friend support was associated with less frequent meal skipping in adolescents at baseline, it was less clear that perceived friend support at this earlier stage was associated with meal skipping frequency in later adolescence. While the results suggested that friends eating healthy foods with them was associated with reduced odds of lunch skipping two years later, this was not the case for breakfast skipping. Furthermore, none of the other measures of perceived friend support were found to reduce the odds of meal skipping at follow-up. On the contrary, friends' discouragement of junk food consumption served to increase the odds of breakfast skipping, an unexpected result, although there was only evidence of increased odds of breakfast skipping for those whose friends sometimes discouraged junk food consumption. While this may be a chance finding as there was no association between this support variable and any other meal-skipping outcomes examined, the avoidance of junk food is a common weight-loss strategy employed during adolescence, as is meal skipping. Therefore, it is possible that encouragement from peers to avoid foods deemed as 'unhealthy' is reflective of a friendship subculture that emphasizes the importance of thinness, joint dieting and restriction ${ }^{(20,23,24,40)}$. This is particularly the case for older adolescent girls, who research demonstrates are more likely to aspire to a thin ideal and engage in extreme weight-loss behaviours ${ }^{(41,42)}$. This might explain why there was only evidence of an association between friends discouraging junk food consumption and breakfast skipping at follow-up. While it is interesting that there was no evidence of an association between discouragement of junk food consumption and lunch skipping at follow-up (for which peer presence is likely), the aforementioned single significant result for this variable was not consistent across time points or meals, further suggesting it was a chance finding.

Given the associations between baseline predictors and meal skipping at follow-up were not consistent with those observed at baseline and were not as clear, it could be the case that friends' support of healthy eating has a more immediate effect on adolescent eating patterns that wanes over time. If this were the case, as the present results would suggest, such influences would be more readily detected in cross-sectional analyses, as opposed to longitudinal analyses conducted over a relatively extended time period. However, as cross-sectional associations at follow-up were similar to the longitudinal associations, one possible explanation for the differences in associations at later age groups may be that factors considered to be important (and therefore influence behaviour) early in life are no longer as important at later life. As adolescents age and become more independent, directly observable behaviours of their peers may have less influence on their own behaviour, as their sense of identity develops ${ }^{(43)}$. Another possible explanation for the less pronounced effect of predictors at follow-up may be that participants had a different friendship group when examined two years later than when first examined at baseline, potentially changing the social environment influencing their behaviour. It is likely that as adolescents age, they develop new interests and personality traits, and as a result may no longer relate to the same friends they had at an earlier developmental stage.

Despite the novel findings of the present study, it was not without its limitations. Perceived friends' encouragement of healthy eating may not reflect reality, as support can be a discrete, non-verbal behaviour that is open to misinterpretation. Similarly, in the present study meal skipping was self-reported and, as with perceived friend support, may have been subject to reporting biases (particularly social desirability) and an increased likelihood of measurement error ${ }^{(44)}$. Future studies should examine friends' support of healthy eating by collecting convergent behavioural data. Further, the reach of the YEP study as a whole was low (46\%). However, this is analogous to that of other large-scale longitudinal studies ${ }^{(44-46)}$ and the sample size in the present study was substantial. Nevertheless, it is important to note that only 1938 participants of the 3264 adolescents who took part in the baseline survey consented to be contacted to participate at follow-up, which may limit the generalizability of these findings. Furthermore, as data were collected from schools located within a single geographic region, external validity is limited, which also has implications for the generalizability of the findings to the wider population. Further research is therefore required to confirm these findings. 
Notwithstanding these limitations, the present study has numerous strengths. Several previously unexamined behaviours measuring perceived friends' support of healthy eating were investigated in relation to adolescent meal skipping. Data were drawn from a large sample of demographically diverse adolescents. The design employed in the current study also allowed for both crosssectional and longitudinal associations to be examined.

\section{Conclusions}

Overall, the present findings suggest that certain socioenvironmental factors that promote healthy eating behaviours may be important to consider when developing nutrition promotion initiatives aimed at reducing unhealthy and disordered eating behaviours in adolescents. Findings suggest adolescents may respond more immediately to directly observable healthy eating behaviours in their friends as opposed to more general encouragements to eat healthy foods or avoid junk foods. Shared beliefs may be the underlying mechanism explaining this association between directly observable dietary behaviour in friends and adolescent meal skipping, as evidenced by the fact that friends eating healthy food appeared to influence meal skipping for meals (i.e. breakfast) eaten at home, where friends' presence is unlikely. Future nutrition promotion strategies should aim to encourage a peer subculture that promotes healthy eating patterns, including the regular consumption of nourishing whole foods.

\section{Supplementary material}

To view supplementary material for this article, please visit https://doi.org/10.1017/S136898001700235X

\section{Acknowledgements}

Acknowledgements: The authors would like to acknowledge the Victorian schools that agreed to participate in the Youth Eating Patterns study. Financial support: This work was supported by the Australian Research Council (grant number DP0452044) and the William Buckland Foundation. K.B. is supported by a National Health and Medical Research Council Principal Research Fellowship (grant number 1042442). The contents of this manuscript are the responsibility of the authors and do not reflect the views of the funding bodies, who had no role in the design, analysis or writing of this article. Conflict of interest: None. Authorship: S.R. carried out the statistical analyses and drafted the paper. K.E.L. was involved in the design and statistical analyses, and contributed to the drafting of the paper. K.B. was involved in the design and conduct of the study, data collection, and contributed to the drafting of the paper. All authors read and approved the final manuscript. Ethics of buman subject participation: The study was approved by Deakin University's Ethics Committee, the Victorian Department of Education and Training, and the Catholic Education Office (EC 227-2003).

\section{References}

1. Serra-Majem L, Ribas L, Pérez-Rodrigo C et al. (2002) Determinants of nutrient intake among children and adolescents: results from the enKid Study. Ann Nutr Metab 46 , 31-38.

2. Story M \& Stang J (2005) Understanding adolescent eating behaviors. In Guidelines for Adolescent Nutrition Services, pp. 9-19 [M Story and J Stang, editors]. Minneapolis, MN: University of Minnesota.

3. Story M, Neumark-Sztainer D \& French S (2002) Individual and environmental influences on adolescent eating behaviors. J Am Diet Assoc 102, 40-51.

4. Alexy U, Wicher M \& Kersting M (2010) Breakfast trends in children and adolescents: frequency and quality. Public Health Nutr 13, 1795-1802.

5. Stea TH, Vik FN, Bere E et al. (2015) Meal pattern among Norwegian primary-school children and longitudinal associations between meal skipping and weight status. Public Health Nutr 18, 286-291.

6. Deshmukh-Taskar PR, Nicklas TA, O'Neil CE et al. (2010) The relationship of breakfast skipping and type of breakfast consumption with nutrient intake and weight status in children and adolescents: the National Health and Nutrition Examination Survey 1999-2006. J Am Diet Assoc 110, 869-878.

7. Hoyland A, Dye L \& Lawton CL (2009) A systematic review of the effect of breakfast on the cognitive performance of children and adolescents. Nutr Res Rev 22, 220-243.

8. Kapantais E, Chala E, Kaklamanou D et al. (2011) Breakfast skipping and its relation to BMI and health-compromising behaviours among Greek adolescents. Public Health Nutr 14, 101-108.

9. Shaw ME (1998) Adolescent breakfast skipping: an Australian study. J Adolesc 33, 851-861.

10. Milosavljević D, Mandić ML \& Banjari I (2015) Nutritional knowledge and dietary habits survey in high school population. Coll Antropol 39, 101-107.

11. Pastore DR, Fisher M \& Friedman SB (1996) Abnormalities in weight status, eating attitudes, and eating behaviors among urban high school students. $J$ Adolesc Health 18, 312-319.

12. Prochnik Estima CdC, da Costa RS, Sichieri R et al. (2009) Meal consumption patterns and anthropometric measurements in adolescents from a low socioeconomic neighborhood in the metropolitan area of Rio de Janeiro, Brazil. Appetite 52, 735-739.

13. Savige G, MacFarlane A, Ball K et al. (2007) Snacking behaviours of adolescents and their association with skipping meals. Int J Behav Nutr Phys Act 4, 36.

14. Rampersaud GC, Pereira MA, Girard BL et al. (2005) Breakfast habits, nutritional status, body weight, and academic performance in children and adolescents. J Am Diet Assoc 105, 743-760.

15. Sjöberg A, Hallberg L, Höglund D et al. (2003) Meal pattern, food choice, nutrient intake and lifestyle factors in the Göteborg Adolescence Study. Eur J Clin Nutr 57, 1569-1578.

16. Pedersen TP, Holstein BE, Flachs EM et al. (2013) Meal frequencies in early adolescence predict meal frequencies in late adolescence and early adulthood. BMC Public Health 13, 445 .

17. Woodruff SJ, Hanning RM, Lambraki I et al. (2008) Healthy Eating Index-C is compromised among adolescents with body weight concerns, weight loss dieting, and meal skipping. Body Image 5, 404-408. 
18. Smith KJ, Gall SL, McNaughton SA et al. (2010) Skipping breakfast: longitudinal associations with cardiometabolic risk factors in the Childhood Determinants of Adult Health Study. Am J Clin Nutr 92, 1316-1325.

19. van Assema P, Glanz K, Martens M et al. (2007) Differences between parents' and adolescents' perceptions of family food rules and availability. J Nutr Educ Behav 39, 84-89.

20. Pearson N, Williams L, Crawford D et al. (2012) Maternal and best friends' influences on meal-skipping behaviours. Br J Nutr 108, 932-938.

21. O'Dea JA (2003) Why do kids eat healthful food? Perceived benefits of and barriers to healthful eating and physical activity among children and adolescents. J Am Diet Assoc 103, 497-501.

22. Contento IR, Williams SS, Michela JL et al. (2006) Understanding the food choice process of adolescents in the context of family and friends. J Adolesc Health 38, 575-582.

23. Eisenberg ME, Neumark-Sztainer D, Story M et al. (2005) The role of social norms and friends' influences on unhealthy weight-control behaviors among adolescent girls. Soc Sci Med 60, 1165-1173.

24. Eisenberg ME \& Neumark-Sztainer D (2010) Friends' dieting and disordered eating behaviors among adolescents five years later: findings from Project EAT. J Adolesc Health 47, $67-73$.

25. Salvy S-J, de la Haye K, Bowker JC et al. (2012) Influence of peers and friends on children's and adolescents' eating and activity behaviors. Physiol Behav 106, 369-378.

26. Pearson N, MacFarlane A, Crawford D et al. (2009) Family circumstance and adolescent dietary behaviours. Appetite 52, 668-674.

27. Woodruff SJ \& Hanning RM (2009) Effect of meal environment on diet quality rating. Can J Diet Pract Res 70, 118-124.

28. Savige GS, Ball K, Worsley A et al. (2007) Food intake patterns among Australian adolescents. Asia Pac J Clin Nutr 16, 738-747.

29. MacFarlane A, Crawford D, Ball K et al. (2007) Adolescent home food environments and socioeconomic position. Asia Pac J Clin Nutr 16, 748-756.

30. Sallis JF, Grossman RM, Pinski RB et al. (1987) The development of scales to measure social support for diet and exercise behaviors. Prev Med 16, 825-836.

31. Keski-Rahkonen A, Kaprio J, Rissanen A et al. (2003) Breakfast skipping and health-compromising behaviors in adolescents and adults. Eur J Clin Nutr 57, 842-853.

32. Mullan B, Wong C \& Kothe E (2013) Predicting adolescent breakfast consumption in the UK and Australia using an extended theory of planned behaviour. Appetite 62, 127-132.
33. Kelder SH, McPherson RS \& Montgomery DH (1996) Meal skipping patterns among children and adolescents. $\mathrm{J} \mathrm{Am}$ Diet Assoc 96, A57.

34. Shi Z, Lien N, Kumar BN et al. (2005) Socio-demographic differences in food habits and preferences of school adolescents in Jiangsu Province, China. Eur J Clin Nutr 59, $1439-1448$.

35. de Castro JM (1994) Family and friends produce greater social facilitation of food intake than other companions. Physiol Behav 56, 445-445.

36. Bruening M, Eisenberg M, MacLehose R et al. (2012) The relationship between adolescents' and their friends' eating behaviors - breakfast, fruit, vegetable, whole grain, and dairy intake. J Acad Nutr Diet 112, 1608-1613.

37. Salvy S-J, Vartanian LR, Coelho JS et al. (2008) The role of familiarity on modeling of eating and food consumption in children. Appetite 50, 514-518.

38. Larson NI, Neumark-Sztainer DR, Story MT et al. (2008) Fast food intake: longitudinal trends during the transition to young adulthood and correlates of intake. $J$ Adolesc Health 43, 79-86.

39. Tang-Péronard JL \& Heitmann BL (2008) Stigmatization of obese children and adolescents: the importance of gender. Obes Rev 9, 522-534.

40. Eapen V, Mabrouk AA \& Bin-Othman S (2006) Disordered eating attitudes and symptomatology among adolescent girls in the United Arab Emirates. Eat Behav 7, 53-60.

41. Wertheim EH, Paxton SJ, Schutz HK et al. (1997) Why do adolescent girls watch their weight? An interview study examining sociocultural pressures to be thin. J Psychosom Res 42, 345-355.

42. Paxton SJ, Schutz HK, Wertheim EH et al. (1999) Friendship clique and peer influences on body image concerns, dietary restraint, extreme weight-loss behaviors, and binge eating in adolescent girls. J Abnorm Psychol 108, 255-266.

43. Marcia JE (1980) Identity in adolescence. In Handbook of Adolescent Psychology, pp. 159-187 [J Adelson, editor]. New York: Wiley.

44. Procidano ME \& Heller K (1983) Measures of perceived social support from friends and from family: three validation studies. Am J Community Psychol 11, 1-24.

45. Neumark-Sztainer D, Eisenberg ME, Fulkerson JA et al. (2008) Family meals and disordered eating in adolescents: longitudinal findings from Project EAT. Arch Pediatr Adolesc Med 162, 17-22.

46. Mikkila V, Rasanen L, Raitakari OT et al. (2004) Longitudinal changes in diet from childhood into adulthood with respect to risk of cardiovascular diseases: the Cardiovascular Risk in Young Finns Study. Eur J Clin Nutr 58, 1038-1045. 